\title{
Indicadores de saúde e aptidão física em escolares do ensino médio da Universidade Federal de Viçosa - Campus Florestal
}

\author{
Indicators of health and physical fitness in schools of average \\ education of Federal University of Viçosa - Campus Florestal
}

Willy Sanches da Silva ${ }^{1}$, Daniel Massote de Melo Leite ${ }^{1}$, Ricardo Wagner de
Mendonça Trigo ${ }^{1}$

1.Universidade Federal de Viçosa - UFV - CAF - Campus Florestal - (MG) - Brasil

\begin{abstract}
Resumo
Introdução: Atualmente o estilo de vida adotado por escolares tem trazido grandes modificações relacionadas à aptidão física para a saúde. A avaliação da aptidão física permite averiguar as condições do indivíduo para melhorar sua capacidade física e qualidade de vida. Objetivo: Analisar as informações sobre as variáveis de composição corporal e aptidão física para a saúde coletados em escolares do Ensino Médio por meio de testes do Manual PROESP-BR. Metodologia: Este estudo caracterizou-se ser de caráter avaliativo qualitativo de dados secundários coletados a partir do PROESP-BR. Para participar do estudo os escolares deveriam ter menos de 18 anos e estarem matriculados na UFV-CAF e possuir atestado médico para participação nas aulas de Educação Física. Foram analisadas as variáveis de aptidão física para a saúde. Resultados: Participaram do estudo 37 estudantes do $1^{\circ}$ ao $3^{\circ}$ ano. Os resultados do IMC apontaram que $(17,85 \%)$ da amostra masculina está em zona de risco para a saúde, contra $(11,11 \%)$ da amostra feminina. O desempenho cardiorrespiratório indicou risco à saúde em $100 \%$ da amostra feminina. No teste de resistência muscular o grupo feminino obteve classificação zona saudável em $100 \%$ da amostra. Em contrapartida, o grupo masculino apresentou valores de risco à saúde $(3,57 \%)$. Na flexibilidade, $100 \%$ da amostra feminina foi classificada em zona saudável. Conclusão: Os escolares apresentaram perfil antropométrico e aptidão física para a saúde satisfatória. A utilização de testes para a avaliação da saúde de escolares foi considerada uma ferramenta útil e deve ser utilizada por professores de Educação Física.
\end{abstract}

Palavras Chave: Escolares; Aptidão física; Saúde. 


\begin{abstract}
Introduction: Today the lifestyle adopted by schoolchildren has brought great modifications related to physical fitness for health. The assessment of physical fitness allows to ascertain the conditions of the individual to improve his physical capacity and quality of life. Objective: To analyze the information on the variables of body composition and physical fitness for health collected in high school students through the PROESP-BR Manual tests. Methodology: This study was characterized as qualitative evaluative character of secondary data collected from PROESP-BR. To participate in the study, students should be under 18 years of age and enrolled in the UFV-CAF and have a medical certificate for participation in Physical Education classes. The variables of physical fitness for health were analyzed. Results: 37 students from 1st to 3rd year participated in the study. The results of the BMI indicated that (17.85\%) of the male sample is in a health risk zone, compared to (11.11\%) in the female sample. Cardiorespiratory performance indicated a health risk in 100\% of the female sample. In the muscular endurance test the female group obtained healthy zone classification in 100\% of the sample. On the other hand, the male group presented values of health risk (3.57\%). In the flexibility, $100 \%$ of the female sample was classified in a healthy zone. Conclusion: Schoolchildren presented an anthropometric profile and physical fitness for satisfactory health. The use of tests to evaluate the health of schoolchildren was considered a useful tool and should be used by Physical Education teachers.
\end{abstract}

Keywords: School children; Physical fitness; Health.

\section{Introdução}

Atualmente o estilo de vida adotado por escolares tem trazido grandes modificações relacionadas à aptidão física para a saúde, influenciando a fase de crescimento e acarretando desvios em sua saúde como desenvolvimento da obesidade, hipertensão arterial, diabetes tipo 2, entre outras doenças metabólicas ${ }^{1-3}$.

Dados da Pesquisa Nacional por Amostra de Domicílios mostram que $62,1 \%$ dos brasileiros com 15 anos ou mais não praticaram qualquer esporte ou atividade física. Sabe-se que 100,5 milhões de pessoas, de um total de 161,8 milhões, nessa faixa etária, não fazem nenhum tipo de exercício físico ${ }^{4}$.

As evidências demonstram que a avaliação dos níveis de aptidão física pode contribuir para a melhora desse prognóstico. Um dos principais componentes de risco para o surgimento de doenças é a baixa aptidão física, a qual é consequência da inatividade física ${ }^{5}$. Devido ao Brasil ser um país com diversas etnias e culturas, fica evidente a importância da descrição e análise de níveis de aptidão física de escolares em diferentes regiões do território brasileiro ${ }^{6}$.

Estudos têm demonstrado a importância de adequados níveis de aptidão física sobre os parâmetros físicos na infância e adolescência para a manutenção de hábitos futuros de atividade física ${ }^{7,8}$. E benefícios para a saúde na adolescência, fase na qual o corpo humano está mais susceptível as modificações no âmbito motor e corporal. Além disso, a mensuração da aptidão física voltada para a saúde em Escolares é fundamental para um bom planejamento do ano letivo e o desenvolvimento da Educação Física Escolar ${ }^{9}$.
A aptidão física é definida como a capacidade de realizar as atividades cotidianas com menor esforço ${ }^{10}$. A aptidão física está relacionada à saúde como condição física nas capacidades que estão profundamente relacionadas, principalmente, à qualidade de vida das pessoas, a flexibilidade, a resistência aeróbia, a força e composição corporal ${ }^{10}$.

Tendo em vista que a avaliação da aptidão física para a saúde pode ser mensuradora de possíveis problemas de saúde, o objetivo deste estudo foi avaliar as variáveis antropométricas e de aptidão física para a saúde de escolares do Ensino Médio Federal da Universidade Federal de Viçosa - CAF e classificá-los de acordo com os padrões propostos pelo Manual de testes do PROESP-BR (2016).

\section{Metodologia}

Tipo de estudo

Este estudo caracterizou-se ser de caráter avaliativo qualitativo de dados secundários coletados, a partir do teste PROESP-BR aplicado em estudantes do Ensino Médio Federal da Universidade Federal de Viçosa - CAF. Esta pesquisa respeitou todas as normas éticas vigentes de pesquisa em seres humanos, conforme Resolução No 466, de dezembro de 2012. O termo de consentimento e assentimento livre esclarecido foi previamente aprovado pela direção de ensino da instituição no momento da inscrição do aluno (a) para a participação nas aulas de Educação Física da UFV- CAF. Para a utilização dos dados, o termo de autorização para a realização da pesquisa foi aprovado e assinado pelo professor regente da turma 
em consenso com a direção da instituição (UFV-CAF12546-6).

\section{Amostra}

A população do estudo foi composta por alunos de ambos os sexos, do $1^{\circ}$ ao $3^{\circ}$ ano, totalizando 37 escolares $(n=37)$, representados por 28 adolescentes do sexo masculino $(\mathrm{N}=28)$ e 9 adolescentes do sexo feminino $(n=9)$. Para participar do estudo, os alunos deveriam ter menos de 18 anos, e estarem matriculados no Ensino Médio Federal. Além disso, deveriam apresentar atestado médico autorizando-os a participar das aulas de Educação Física.

\section{Delineamento experimental}

A coleta de dados foi obtida por meio dos resultados da aplicação do manual de testes escolares do PROESP-BR (2016), no início do ano letivo. O manual de testes PROESP-BR é um sistema de avaliação da aptidão física relacionada à saúde e ao desempenho esportivo de crianças e adolescentes no âmbito da Educação Física escolar e do esporte educacional $^{11}$. Com a finalidade de operacionalizar a aplicação do PROESP-BR pelo professor durante as aulas de Educação Física, sugerimos dividir a bateria de testes ao longo de doze aulas, em três semanas.

Nas quatro primeiras aulas, foram aplicados as medidas de massa corporal $(\mathrm{Kg})$, estatura (cm) (IMC) e o teste de flexibilidade "sentar e alcançar"; nas quatro aulas da semana seguinte, aplicaram-se os testes de resistência muscular localizada, abdominais ( sit up) e, na terceira semana de aulas, foi aplicado o teste de aptidão cardiorrespiratória corrida/caminhada de 6 minutos.

Para a coleta de medidas antropométricas, utilizamos as variáveis de peso e altura de acordo com o protocolo proposto pelo PROESP-BR (2016). A medida de massa corporal $(\mathrm{Kg})$ e altura $(\mathrm{Cm})$ foram mensuradas utilizando uma balança clínica profissional (precisão em $100 \mathrm{~g}$ ) e estadiômetro (precisão em 0,5 cm) (WELMY, SÃO PAULO, BRASIL). Os alunos foram orientados a vestir preferencialmente trajes de Educação Física e ficarem descalços. O Índice de Massa Corporal (IMC) foi calculado utilizando a fórmula $\left(\mathrm{Kg} / \mathrm{m}^{2}\right)$ e os alunos foram classificados de acordo com o grau de adiposidade dos pontos de corte de IMC adotados pelo manual do PROESP-BR ${ }^{11}$.

\section{Teste de flexibilidade}

Para a avaliação da (Flex) flexibilidade dos alunos, foi utilizado o teste de sentar e alcançar, foi estendida uma fita métrica no solo. Na marca de $38 \mathrm{~cm}$ desta fita, foi colocado um pedaço de fita adesiva de $30 \mathrm{~cm}$ em perpendicular. A fita adesiva deve fixar a fita métrica no solo. O sujeito avaliado deveria estar descalço. Os calcanhares deveriam tocar a fita adesiva na marca dos 38 centímetros e ficarem separados por 30 centímetros. Com os joelhos estendidos e as mãos sobrepostas, o avaliado inclina-se lentamente e estende as mãos para frente o mais distante possível. $\mathrm{O}$ avaliado deve permanecer nesta posição o tempo necessário para a distância ser anotada. Foram realizadas duas tentativas por aluno (a). O resultado é medido em centímetros, a partir da posição mais longínqua que o aluno pode alcançar na escala com as pontas dos dedos. Foram registrados os resultados com uma casa após a vírgula. Para a avaliação, foram analisados os melhores resultados em duas tentativas consecutivas $^{11}$.

\section{Teste de resistência muscular localizada}

Para a avaliação da resistência muscular, foi utilizado o teste Sit - up. O aluno avaliado se posiciona em decúbito dorsal com os joelhos flexionados a 45 graus e com os braços cruzados sobre o tórax. $\mathrm{O}$ avaliador, com as mãos, segura os tornozelos do estudante fixando-os ao solo. Ao sinal, o aluno iniciava os movimentos de flexão do tronco até tocar com os cotovelos nas coxas, retornando à posição inicial, não tocando, necessariamente, com a cabeça no colchonete a cada execução. O protocolo consiste em realizar o maior número de repetições completas em 1 minuto. O resultado anotado é expresso pelo número de movimentos completos realizados em 1 minuto ${ }^{11}$.

Teste de aptidão cardiorrespiratória corrida/caminhada de 6 minutos.

O teste de aptidão cardiorrespiratória foi realizado em uma pista de atletismo. Informamos aos alunos sobre a execução do teste, dando ênfase ao fato de que deviam correr o maior tempo possível, evitando piques de velocidade intercalados por longas caminhadas. Durante o teste, informamos ao aluno a passagem do tempo 2', 4' e 5' (“Atenção: falta 1 minuto). Ao final do teste um sinal (apito) foi dado. Os alunos deveriam interromper a corrida, permanecendo no lugar onde estavam (no momento do apito) até ser anotada ou sinalizada a distância percorrida. Os resultados foram anotados em metros com uma casa após a vírgula ${ }^{11}$.

\section{Análise estatística}

Os dados foram tabulados e a análise descritiva dos dados (mínimo, máximo, média e desvio padrão) foram feitas pelo software SIGMAPLOT 11.0. Todos os dados de aptidão física para a saúde foram classificados e realizadas as respectivas frequências de cada grupo para a caracterização dos pontos de corte em zona de risco à saúde; com valores abaixo, e zona saudável, com valores acima de acordo com o manual do PROESP-BR ${ }^{11}$.

\section{Resultados}

O estudo incluiu 37 escolares $(n=37)$ da Universidade Federal de Viçosa - CAF, 28 adolescentes do sexo masculino e 9 adolescentes do sexo feminino. Características e análise descritiva das 
variáveis, idade, estatura, massa corporal, IMC e dos testes de aptidão física da amostra do sexo feminino e masculino estão descritos na tabela 1 . Os resultados do IMC e massa corporal total do presente estudo no grupo feminino $(n=9)$ apresentaram menores valores médios de IMC $(19,66 \mathrm{~kg} / \mathrm{m} 2)$ e massa corporal $(48,48$ $\mathrm{Kg})$ em relação ao grupo masculino $(\mathrm{n}=28)$ que apresentou maiores valores médios de IMC (20,76 $\mathrm{kg} / \mathrm{m} 2)$ e massa corporal $(60,33 \mathrm{Kg})$. Os resultados de massa corporal e IMC diferentes entre os grupos demonstram que mudanças na composição corporal entre meninos e meninas podem estar relacionados ao período púbere. Esses achados apresentam limitações, devido a não avaliação do estadiamento puberal em ambas as amostras, que podem interferir em modificações antropométricas da composição corporal.

Observando a análise descritiva das variáveis de aptidão física para a saúde, podemos verificar que os alunos do sexo feminino apresentaram menores valores de média no teste de caminhada/corrida (791,1 $\mathrm{m})$, em relação ao grupo masculino $(1261,6 \mathrm{~m})$ e média superior que o grupo masculino na flexibilidade $(45,5$ $\mathrm{cm}$ vs $42,1 \mathrm{~cm})$ e inferior no teste de resistência abdominal $(38,1 \mathrm{r} / \mathrm{min}$ vs $46,0 \mathrm{r} / \mathrm{min})$ do grupo masculino.

TABELA 1 - Análise descritiva das variáveis, idade, estatura, massa corporal, IMC e dos testes de aptidão física da amostra do sexo feminino e masculino.

\begin{tabular}{cccccc}
\hline $\begin{array}{c}\text { Variáveis } \\
\text { (Feminino) }\end{array}$ & $\mathrm{N}$ & Mínimo & Máximo & Média & Desvio padrão \\
Idade & 9 & 15,00 & 17,00 & 16,00 & 0,70 \\
Estatura (metros) & 9 & 1,46 & 1,65 & 1,57 & 0,05 \\
Massa corporal & 9 & 37,00 & 62,00 & 48,48 & 7,12 \\
$\quad$ (Kg) & 9 & 17,40 & 24,80 & 19,66 & 2,15 \\
IMC & 9 & 675,00 & 885,00 & 791,11 & 83,99 \\
Caminhada 6 (min) & 9 & 31,00 & 53,00 & 45,55 & 7,63 \\
Flexibilidade & 9 & 24,00 & 52,00 & 38,11 & 9,74 \\
Sit-up (Abdominal) & 9 & &
\end{tabular}

$\begin{array}{cccccc}\begin{array}{c}\text { Variáveis } \\ \text { (Masculino) }\end{array} & \mathrm{N} & \text { Mínimo } & \text { Máximo } & \text { Média } & \text { Desvio padrão } \\ \text { Idade } & 28 & 14,00 & 17,00 & 15,64 & 1,06 \\ \text { Estatura (metros) } & 28 & 1,57 & 1,83 & 1,69 & 0,06 \\ \begin{array}{c}\text { Massa corporal } \\ \text { (kg) }\end{array} & 28 & 40,00 & 113,00 & 60,35 & 15,00 \\ \text { IMC } & 28 & 14,50 & 33,70 & 20,82 & 4,16 \\ \text { Caminhada 6 (min) } & 28 & 970,00 & 1550,00 & 1261,60 & 148,14 \\ \quad \text { Flexibilidade } & 28 & 20,00 & 70,00 & 42,17 & 11,67 \\ \text { Sit-up (Abdominal) } & 28 & 28,00 & 72,00 & 46,00 & 9,39\end{array}$


Quanto ao diagnóstico do IMC e dos testes de aptidão física para a saúde, apresentamos os dados neste estudo por meio da frequência e classificação baseados em quadros classificatórios disponíveis no manual de testes do PROESP-BR, a interpretação dos valores está acima dos pontos de corte como zona saudável e os valores abaixo como zona de risco à saúde ${ }^{12}$. Na tabela 2, foram expressos os valores críticos de IMC para a saúde, frequência e classificação do grupo feminino e masculino. Podemos observar que $11,11 \%=1$ da amostra total de 16 anos do grupo feminino apresentou valores acima do ponto de corte $(>24,0)$ indicando risco à saúde. As idades de 15 e 17 anos apresentaram valores abaixo do ponto de corte, indicando zona saudável. No grupo masculino a idade de 14 anos $(3,57 \%=1), 15$ anos
$(7,14 \%=2)$ e 17 anos $(7,14=2)$ apresentaram risco a saúde, ficando acima do ponto de corte. A idade de 16 anos mostrou valores abaixo do ponto de corte $(>24,0)$, indicando zona de saúde saudável. Fatores nutricionais, nível de atividade física, diferenças hormonais não foram avaliadas em nossa pesquisa, e podem explicar a frequência maior de risco à saúde pelo IMC no sexo masculino. Uma das limitações do estudo foi a não realização da avaliação física por dobras cutâneas, uma vez que o índice de massa corporal indica a massa corporal total, não separando a massa muscular, esqueleto axial, apendicular e percentual de gordura $^{12,13}$. Isso pode interferir nos resultados encontrados.

TABELA 2 - Idade, valores críticos de IMC para a saúde, frequência e classificação do grupo masculino e

\begin{tabular}{ccccc}
\hline $\begin{array}{c}\text { Idade } \\
\text { (Feminino) }\end{array}$ & Valores Críticos* & $\mathrm{n}$ & $\%$ & Classificação** \\
$\mathbf{1 5}$ & 22,4 & 2 & 22,23 & Zona Saudável \\
$\mathbf{1 6}$ & 24,0 & 4 & 44,46 & Zona Saudável \\
$\mathbf{1 6}$ & 24,0 & 1 & 11,11 & Zona de Risco \\
$\mathbf{1 7}$ & 24,0 & 2 & 22,22 & Zona Saudável \\
- & - & $\Sigma$ & 100,00 & -
\end{tabular}

$\begin{array}{ccccc}\begin{array}{c}\text { Idade } \\ \text { Masculino) }\end{array} & \text { Valores Críticos* } & \mathrm{n} & \% & \text { Classificação** } \\ \mathbf{1 4} & 22,2 & 3 & 10,71 & \text { Zona Saudável } \\ \mathbf{1 4} & 22,2 & 1 & 3,57 & \text { Zona de Risco } \\ \mathbf{1 5} & 23,0 & 8 & 28,57 & \text { Zona Saudável } \\ \mathbf{1 5} & 23,0 & 2 & 7,14 & \text { Zona de Risco } \\ \mathbf{1 6} & 24,0 & 6 & 21,42 & \text { Zona Saudável } \\ \mathbf{1 7} & 25,4 & 6 & 21,42 & \text { Zona Saudável } \\ \mathbf{1 7} & 25,4 & 2 & 7,14 & \text { Zona de Risco } \\ \mathbf{-} & - & \sum & 100,00 & \text { - }\end{array}$

\footnotetext{
PROESP-BR (2016).

A tabela 3 apresenta a frequência e classificação da aptidão cardiorrespiratória do sexo feminino e masculino. No grupo feminino, todas as alunas $(n=9)$ obtiveram baixo desempenho cardiorrespiratório, indicando risco à saúde em $100 \%$ da amostra, independentemente da idade. No grupo masculino, foi encontrada baixa classificação relacionada ao risco à saúde, devido à baixa frequência no grupo de 15 anos $(10,71 \%=3), 16 \operatorname{anos}(3,57 \%=1)$ e $17 \operatorname{anos}(3,57 \%=1)$. Os resultados insatisfatórios do teste
}

*Valores críticos apresentados e ** classificação de ambos os grupos apresentados como referência do manual do

caminhada/corrida podem ser verificados em outros estudos conforme Silva et al. (2010) ${ }^{14}$, que indicou fatores culturais, motivacionais e estruturais de cunho motor e distribuição corporal, podem influenciar negativamente as meninas em relação aos meninos. Podemos inferir, que em nosso estudo, verificamos baixo fator motivacional para a realização do teste, devido a fatores ambientais e falta de interesse. Podemos citar que a boa aptidão cardiorrespiratória contra a inatividade física pode evitar problemas futuros, como doenças cardíacas, complicações motoras e sedentarismo. 
TABELA 3 - Frequência e classificação da aptidão cardiorrespiratória do sexo feminino e masculino.

\begin{tabular}{|c|c|c|c|c|}
\hline $\begin{array}{c}\text { Idade } \\
\text { (Feminino) }\end{array}$ & Valores Críticos* & $\mathrm{n}$ & $\%$ & Classificação** \\
\hline $\begin{array}{c}15 \\
16 \\
17 \\
-\end{array}$ & $\begin{array}{c}1005 \\
1070 \\
1110 \\
-\end{array}$ & $\begin{array}{l}2 \\
5 \\
2 \\
\sum\end{array}$ & $\begin{array}{c}22,22 \\
55,56 \\
22,22 \\
100\end{array}$ & $\begin{array}{l}\text { Zona de Risco } \\
\text { Zona de Risco } \\
\text { Zona de Risco }\end{array}$ \\
\hline $\begin{array}{c}\text { Idade } \\
\text { (Masculino }\end{array}$ & Valores Críticos* & $\mathrm{n}$ & $\%$ & Classificação** \\
\hline 14 & 1060 & 4 & 14,02 & Zona saudável \\
\hline 15 & 1130 & 7 & 25,0 & Zona saudável \\
\hline 15 & 1130 & 3 & 10,71 & Zona de risco \\
\hline 16 & 1190 & 5 & 17,85 & Zona saudável \\
\hline 16 & 1190 & 1 & 3,57 & Zona de risco \\
\hline 17 & 1190 & 7 & 25,0 & Zona saudável \\
\hline 17 & 1190 & 1 & 3,57 & Zona de risco \\
\hline- & - & $\sum$ & 100 & \\
\hline
\end{tabular}

*Valores críticos apresentados e ** classificação de ambos os grupos apresentados como referência do manual do PROESP-BR (2016).

Quando analisamos a tabela 4, podemos observar que o grupo feminino obteve classificação zona saudável em $100 \%$ da amostra total no teste de resistência muscular localizada. $\mathrm{O}$ grupo masculino apresentou valores de risco à saúde nas idades de 14 , 15 e 17 anos $(3,57 \%=1)$. A melhora da resistência muscular como encontrado no grupo feminino, está associada a uma redução das doenças crônicas e melhora no desempenho de atividades físicas, o equilíbrio postural e alinhamento de segmentos corporais que têm influência significante na musculatura abdominal e seu fortalecimento pode prevenir possíveis lesões na coluna lombar ou possíveis hérnias abdominais, logo essa musculatura fortalecida permite a estabilização dessa região ${ }^{15}$.

TABELA 4 - Frequência e classificação de resistência muscular localizada do sexo feminino e masculino.

$\begin{array}{ccccc}\begin{array}{c}\text { Idade } \\ \text { (Feminino) }\end{array} & \text { Valores Críticos* } & \mathrm{n} & \% & \text { Classificação** } \\ & & & & \\ \mathbf{1 5} & 23,0 & 2 & 22,22 & \text { Zona saudável } \\ \mathbf{1 6} & 23,0 & 5 & 55,56 & \text { Zona saudável } \\ \mathbf{1 7} & 23,0 & 2 & 22,22 & \text { Zona saudável } \\ \mathbf{-} & - & \sum & 100 & \end{array}$

\begin{tabular}{ccccc}
$\begin{array}{c}\text { Idade } \\
\text { (Masculino }\end{array}$ & Valores Críticos* & $\mathrm{n}$ & $\%$ & Classificação** \\
) & & & & \\
$\mathbf{1 4}$ & 35,0 & 3 & 10,71 & Zona saudável \\
$\mathbf{1 4}$ & 35,0 & 1 & 3,57 & Zona de risco \\
$\mathbf{1 5}$ & 35,0 & 9 & 32,14 & Zona saudável \\
$\mathbf{1 5}$ & 35,0 & 1 & 3,57 & Zona de risco \\
$\mathbf{1 6}$ & 40,0 & 6 & 21,42 & Zona saudável \\
$\mathbf{1 7}$ & 40,0 & 7 & 25,00 & Zona saudável \\
$\mathbf{1 7}$ & 40,0 & 1 & 3,57 & Zona de risco \\
$\mathbf{-}$ & - & $\sum$ & 100 & \\
\hline
\end{tabular}

*Valores críticos apresentados e ** classificação de ambos os grupos apresentados como referência do manual do PROESP-BR (2016). 
Outro componente avaliado, a flexibilidade, (tabela 5) apresentou frequência de $100 \%$ do total da amostra feminina na classificação zona saudável. No grupo masculino, apenas $3,57 \%=1$ da amostra de 15 anos apresentou risco à saúde. As demais idades de 16 anos e 17 anos apresentaram valores similares 3,57\% = 1 da amostra com risco à saúde. Nossos resultados demonstram que ambos os grupos apresentaram níveis satisfatórios de flexibilidade. Alguns estudos na literatura demonstraram a existência de diferença de flexibilidade muscular entre os sexos em adultos, a mulher é mais flexível que o homem ${ }^{16,17}$. São escassos estudos que avaliaram quais fatores contribuem para uma menor flexibilidade de adolescentes de ambos os sexos. Estudos mostram que a flexibilidade muscular se relaciona com fatores genéticos, estilo de vida, sexo e idade ${ }^{18}$.

\section{TABELA 5 -Freq uência e classifica ção de flexibilidade do sexo feminino e masculino.}

\begin{tabular}{ccccc}
\hline $\begin{array}{c}\text { Idade } \\
\text { (Feminino) }\end{array}$ & Valores Criticos * & $\mathrm{n}$ & $\%$ & Classificação** \\
& & & & \\
$\mathbf{1 5}$ & 24,3 & 2 & 22,22 & Zona saudável \\
$\mathbf{1 6}$ & 24,3 & 5 & 55,56 & Zona saudável \\
$\mathbf{1 7}$ & 24,3 & 2 & 22,22 & Zona saudável \\
- & - & $?$ & 100 & \\
\hline
\end{tabular}

$\begin{array}{ccccc}\begin{array}{c}\text { Idade } \\ \text { Masculino }\end{array} & \text { Val ores Criticos * } & \text { n } & \% & \text { Classificação** } \\ \mathbf{1 4} & 22,9 & 4 & 14,02 & \\ \mathbf{1 5} & 24,3 & 9 & 32,14 & \text { Zona saudável } \\ \mathbf{1 5} & 24,3 & 1 & 3,57 & \text { Zona saudável } \\ \mathbf{1 6} & 25,7 & 5 & 17,85 & \text { Zona de risco } \\ \mathbf{1 6} & 25,7 & 1 & 3,57 & \text { Zona saudável } \\ \mathbf{1 7} & 25,7 & 7 & 25,0 & \text { Zona de risco } \\ \mathbf{1 7} & 25,7 & 1 & 3,57 & \text { Zona saudável } \\ - & - & ? & 100 & \text { Zona de risco } \\ \end{array}$

*Valores críticos apresentados e ** classificação de ambos os grupos apresentados como referência do manual do PROESP-BR (2016).

\section{Discussão}

De acordo com os resultados encontrados nas análises das variáveis do respectivo estudo, a maioria dos alunos (as) do Ensino Médio Federal da UFV-CAF apresentam ter baixo risco à saúde, indicando satisfatória aptidão física para a saúde segundo as diretrizes do manual do PROESP-BR ${ }^{11}$. Este estudo mostrou resultados contrários com a literatura atual que demonstra falta de interesse dos estudantes nas aulas de Educação Física, prática esportiva, nos hábitos de lazer, e no aumento excessivo de horas diárias diante de diversos aparelhos eletrônicos ${ }^{19-21}$, demonstrando níveis insatisfatórios de aptidão física para a saúde. Em nosso estudo, não avaliamos níveis de atividade física, hábitos de lazer e número de horas diárias diante de aparelhos tecnológicos atuais, como celulares ou computadores, podendo ser uma limitação do estudo. Podemos inferir, que a localidade de estudo (UFV-CAF) possui ampla infraestrutura esportiva e uma gama de possibilidades de prática esportiva, em horários alternativos e durante as aulas de Educação Física, contribuindo para os resultados encontrados.

A mensuração do peso e altura, associado à idade permite avaliar o estado nutricional da população alvo, que é de suma importância para analisar a saúde e a condição de vida. O IMC (calculado através da divisão do peso em $\mathrm{kg}$ pela altura em metros elevada ao quadrado, $\mathrm{kg} / \mathrm{m}^{2}$ ) é o cálculo mais usado para avaliação da adiposidade corporal ${ }^{22}$. A prevalência de obesidade e sobrepeso em adolescentes brasileiros tem chamado a atenção dos cientistas ${ }^{23}$. Em nosso estudo os resultados da frequência e classificação do IMC apontaram que os alunos $(17,85 \%)$ da amostra estão em zona de risco a saúde em relação as alunas $(11,11 \%)$. Esses resultados colaboram com outros estudos feitos em outros escolares brasileiros. Estudo de Farias Júnior et al. $^{24}$ em escolares brasileiros utilizando o IMC, mostrou que a prevalência de sobrepeso/obesidade na cidade de João Pessoa-PB foi de $10 \%$, mostrando-se mais elevada nos meninos do que nas meninas $(13,5 \%$ vs $7,4 \%)$. Resultados semelhantes também encontrados por Carneiro et al. 
$(2017)^{25}$ demonstram que o excesso de peso foi significativamente mais prevalente no sexo masculino do que no sexo feminino (26,3 versus $16,8 \%$ ). Fatores nutricionais, relação socioeconômica, diferenças hormonais e puberdade, que não foram avaliados em nossa pesquisa, podem explicar a frequência maior de risco à saúde no sexo masculino. Além desses fatos, a amostra pode ser um fator preponderante nos resultados do estudo. O grupo feminino contou com nove voluntários $(n=9)$ e o grupo masculino vinte e oito voluntários $(n=28)$. Guedes e colaboradores especulam que diferenças quanto ao tamanho das amostras, à seleção dos sujeitos e aos instrumentos de medidas empregados na identificação dos níveis de prática de atividade física habitual e dos índices de aptidão física possam justificar as divergências observadas $^{26}$.

Os testes de aptidão física relacionada à saúde compreendem as análises de resistência cardiorrespiratória, resistência muscular localizada e flexibilidade e composição corporal ${ }^{11}$. Resultados insatisfatórios em testes de aptidão para a saúde podem afetar todos os sistemas do corpo, diminuindo sua tolerância ao estresse fisiológico em resposta ao exercício físico e a doenças crônicas, causando comorbidades, incluindo desenvolvimento de diabetes mellitus tipo 2 (DM 2), dislipidemias, hipertensão, puberdade precoce, irregularidades menstruais, apneia do sono, asma, distúrbios musculoesqueléticos, problemas psicológicos dentre outros, que estão associadas a uma vida sedentária ${ }^{27}$.

No teste de resistência cardiorrespiratória, as meninas obtiveram baixo desempenho cardiorrespiratório, indicando risco à saúde em $100 \%$ da amostra independentemente da idade. Resultados inadequados encontrados em adolescentes brasileiros em testes de aptidão cardiorrespiratória podem ser vistos em outros estudos ${ }^{18}$. Conforme Carissimi et al. ${ }^{28}$ e Silva et al. ${ }^{14}$ fatores culturais, motivacionais e estruturais de cunho motor e distribuição corporal, podem influenciar negativamente a participação das alunas em testes de aptidão física em relação aos alunos. Podemos inferir que, nos resultados do estudo, verificamos baixo fator motivacional para a realização do teste devido a fatores ambientais (calor e raios solares) e falta de interesse por parte das alunas, com a elevação dos níveis de sudorese e desconforto durante $\mathrm{o}$ teste. Pesquisadores que encontraram resultados insatisfatórios em escolares nos níveis de aptidão cardiorrespiratória Burgos et al. ${ }^{29} \mathrm{e}$ Silva et al. ${ }^{14}$ têm demonstrado preocupação com essa população. Revisão sistemática identificou que adolescentes do sexo feminino, de nível econômico baixo, com níveis insuficientes de atividade física, sedentários e com excesso de adiposidade corporal foram subgrupos de risco para apresentarem baixa aptidão cardiorespiratoria ${ }^{30}$. No Brasil, a maioria dos estudos apontou que cerca de $80 \%$ dos adolescentes apresentaram baixos níveis de aptidão cardiorrespiratória para a saúde ${ }^{31,32}$ devido à inatividade física. Diferentemente da literatura atual, o grupo masculino apresentou menor classificação de risco à saúde no grupo de 15 anos 16 e 17 anos. Apesar disso, resultados contrários a este estudo foram encontrados. Estudo transversal recente realizado por Pelegrini et al. ${ }^{33}$ com 601 escolares brasileiros de 14 a 17 anos de idade, apontou uma prevalência de aptidão cardiorrespiratória inadequada de $61.1 \%$ no grupo do sexo masculino. Nesse contexto, visto que, boa aptidão física para a saúde pode evitar problemas futuros relacionados a saúde. ${ }^{34,35}$ os resultados de aptidão cardiorrespiratória apresentam boas perspectivas para os escolares do sexo masculino.

A flexibilidade é uma das características do sistema musculoesquelético que promove melhor eficiência de movimentos diários, melhora o desempenho muscular em atividades físicas e influência positivamente a postura do indivíduo, além de prevenir algumas patologias na coluna vertebral. $^{23,18}$

Os resultados do teste de flexibilidade apresentaram frequência de $100 \%$ do total da amostra feminina na classificação zona saudável. No grupo masculino 3,57\% da amostra apresentou classificação em zona de risco à saúde. Podemos verificar que as meninas possuem maiores níveis de flexibilidade quando comparado aos meninos, no entanto, não existe um consenso na literatura sobre qual sexo apresenta ser mais flexível na fase da infância e adolescência. Novas pesquisas precisam ser realizadas para verificar níveis de atividade física, tipo de esporte praticado entre outros fatores. Os estudos encontrados mostram diferenças positivas de flexibilidade em mulheres adultas em relação aos homens adultos ${ }^{16,17}$. Dados encontrados em outras pesquisas com idades inferiores, afirmaram com seus resultados que dos 5 aos 10 anos, as meninas são mais flexíveis que os $\operatorname{meninos}^{37,38}$. Diversos fatores podem estar relacionados com os achados do presente estudo, fatores genéticos, sexo e idade ${ }^{18,39}$, membros inferiores desproporcionais em relação ao tronco, comprimento de fibras musculares, postura e abdução escapular inadequada podem influenciar nos resultados do teste de flexibilidade. Estudos com resultados similares em idades semelhantes foram achados na literatura. Fernandes et al..$^{40}$ e Mikkelson et al ${ }^{18}$ encontraram melhor desempenho no teste de flexibilidade em meninas. Mikkelson et al. ${ }^{18}$ encontraram valores médios no teste de sentar-e-alcançar de $56,8 \pm 7,5 \mathrm{~cm}$ em meninos e $60,9 \pm 6,1 \mathrm{~cm}$ em meninas com idades entre 12 e 17 anos.

A resistência muscular é um importante componente da aptidão física e permite realizar melhor as tarefas diárias. A melhora da resistência muscular está associada a uma redução de doenças crônicas e melhora no desempenho de atividades físicas ${ }^{41,42}$. Além disso, o equilíbrio postural e alinhamento de segmentos corporais têm influência significante na musculatura abdominal e seu fortalecimento pode prevenir possíveis lesões na coluna lombar ou possíveis hérnias abdominais, logo essa musculatura fortalecida permite a estabilização dessa região ${ }^{43}$. A resistência muscular localizada avaliada pelo teste sit 
up apontou que o grupo feminino obteve classificação zona saudável em $100 \%$ da amostra total enquanto o grupo masculino apresentou valores de risco à saúde nas idades de 14, 15 e 17 anos. Tem sido demonstrado que adolescentes apresentam baixos níveis de resistência abdominal. Estudo que avaliaram escolares mostrou que 98,4\% dos adolescentes de ambos os sexos, não atenderam aos critérios mínimos estabelecidos para saúde no teste de abdominais modificado em 1 minuto ${ }^{44}$. Temos a limitação da amostra com número menor no grupo feminino, o que pode influenciar nos resultados. Apesar disso, resultados similares em nossas análises foram achados por Mascarenhas et al., ${ }^{43}$ em idades inferiores, com $73,3 \%$ dos escolares classificados em zona de risco à saúde. Inversamente encontrado no presente estudo, Luguetti et al. ${ }^{45}$ apresentou a classificação do teste de resistência abdominal por porcentagem em 3145 escolares de 7 a 16 anos da rede pública da cidade de São Paulo. Em média, os piores valores foram de $40 \%$ das meninas e $36 \%$ dos meninos na classificação do seu estudo.

É importante destacar que o presente estudo apresenta algumas limitações, como a não mensuração do volume e intensidade das atividades físicas praticadas pelos estudantes fora do ambiente escolar; da frequência de participação nas aulas e do nível de motivação dos alunos; aspectos relevantes na determinação do nível de atividade física e de seus efeitos na aptidão física para a saúde.

Entretanto, futuramente, é necessária uma investigação mais detalhada, com maior número e igualdade de estudantes entre ambos os sexos, avaliação dos conteúdos e estudos pós-intervencionais das aulas de Educação Física Escolar para aprofundamento do tema.

Por fím, após a avaliação de todos os parâmetros de aptidão física para a saúde, os estudantes apresentaram boa aptidão física, dessa forma, as aulas de Educação Física Escolar, respeitando todos os parâmetros e individualidades, não impedem maior complexidade e exigência dos alunos.

\section{Conclusão}

O objetivo deste estudo foi avaliar as variáveis antropométricas e de aptidão física para a saúde de escolares do Ensino Médio Federal da Universidade Federal de Viçosa - CAF e classificá-los de acordo com os padrões propostos pelo Manual de testes do PROESP-BR (2016).

Os principais resultados do presente estudo mostraram que os escolares apresentaram perfil antropométrico e aptidão física para a saúde satisfatória, dentro dos padrões propostos pelo PROESP-BR. O grupo feminino por seus componentes e estruturas corporais apresentaram boa flexibilidade, e foram classificados em zona saudável para a saúde. O grupo masculino obteve bons resultados no teste de força abdominal e teste corrida/caminhada, indicando zona saudável a saúde, esse resultado é um importante fator protetor contra doenças cardiovasculares. $\mathrm{O}$ grupo masculino apresentou média de IMC e massa corporal mais elevada do que o grupo feminino, a idade de 14 anos, 15 anos e 17 anos apresentou risco a saúde, ficando acima dos valores críticos propostos pelo manual do PROESP-BR: 22,2 para idade de 14 anos; 23,0 para a idade de 15 anos e 25,4 para a idade de 17 anos respectivamente. Apenas a idade de 16 anos mostrou valores abaixo dos padrões críticos 24,0 para a idade de 16 anos, indicando zona saudável para a saúde.

Com esses resultados, podemos confirmar que a utilização de testes para aptidão física para a avaliação da saúde de escolares é considerada uma ferramenta útil e barata e deve ser utilizada por professores de Educação Física para avaliação de seus estudantes no intuito de oferecer uma intervenção mais precisa e promover a melhora da aptidão física com atividades físicas.

Com base nos dados do estudo, podemos inferir que os escolares estudados diferem da realidade atual da aptidão física mundial, onde são encontrados altos níveis de inatividade física, obesidade e consequentemente sedentarismo, que podem levar a distúrbios na saúde de adolescentes na fase adulta.

\section{Declaração de conflitos de interesses}

Os autores do artigo afirmam que não houve nenhuma situação de conflito de interesse, tais como propostas de financiamento, emissão de pareceres, promoções ou participação em comitês consultivos ou diretivos, entre outras, que pudessem influenciar no desenvolvimento do trabalho.

\section{Referências}

1.Silva, L. V. M. Associação entre variáveis de composição corporal e aptidão física em adolescentes. Revista de Atenção à Saúde, v. 12, n. 41, p.51-56, jul./set. 2014.

2. Leblanc, A. G.; Katzmarzyk, P. T.; Barreira, T. V.; Broyles, S. T.; Chaput, J. P.; Church, T. S.; Fogelholm, M.; Harrington, D. M.; Hu, G.; Kuriyan, R.; Kurpad, A.; Lambert, E. V.; Maher, C.; Maia, J.; Matsudo, V.; Olds, T.; Onywera, V.; Sarmiento, O.L.; Standage, M.; Tudor-Locke, C.; Zhao, P.; Tremblay, M.S.; Iscole Research Group. Correlates of total sedentary time andscreen time in 9-11 year-old children around the world: the international study of childhood obesity, lifestyle and the environment. PlosOne, v. 10, n. 6. 2015.

3. Schubertl, A., Januário, R.S.B., Casonatto J., Sonoo C. N. Aptidão física relacionada à prática esportiva em crianças e adolescentes. Rev Esp Med Esporte,v. 22, n.2, p.142-6. 2016.

4. Soares, B. C. Pesquisa Nacional de Saúde do Escolar: Instituto Brasileiro de Geografia e Estatística. 
3. ed. Rio de Janeiro: Centro de Documentação e Disseminação de Informações, 2015

5. De Sousa, E. P; Moreira, O. C. Importância da aptidão física relacionada à saúde e aptidão motora em crianças e adolescentes. RBPFEX-Revista Brasileira de Prescrição e Fisiologia do Exercício, v. 7, n. 39, 2013.

6. Pereira C. H, Ferreira D. S, Copetti G. L, Guimarães L. C, Barbacena M. M, Liggeri N, Castro O. G, Lobato S, David A. C. Aptidão física em escolares de uma unidade de ensino da rede pública de Brasília Distrito Federal. Revista Brasileira de Atividade Física e Saúde, v. 16, n.3, p. 223-227, 2011.

7. Cleland V, Dwyer T, Venn A. Which domains of childhood physical activity predict physical activity in adulthood? A 20-year prospective tracking study. Br J Sports Med, v. 46, n.8, p. 595-602. 7. 2012.

8. Balassiano D. H, Araújo C. G. Frequência cardíaca máxima: influência da experiência desportiva na infância e adolescência. Arq Bras Cardiol. V 100(4), p. 333-8. 2013.

9. Farias, E. D. S., Carvalho, W. R. G. D., Gonçalves, E. M., \& Guerra-Júnior, G. Efeito da atividade física programada sobre a aptidão física em escolares adolescentes. Revista Brasileira de Cineantropometria \& Desempenho Humano, 2010.

10. Oliveira, R. R.; Santos, M. G. Componentes da aptidão física relacionada à saúde. Revista Digital Efdeportes.com, Buenos Aires, p.01-06, jun, 2012.

11. Gaya, A., Gaya, A. Projeto Esporte Brasil PROESP-Br: Manual de testes e avaliação. Ufrgs, Porto Alegre, p.01-25, 2016.

12. Garn, S. M., Leonard, W. R., \& Hawthorne, V. M. (1986). Three limitations of the body mass index, 1986.

13. Rech, C. R., Petroski, E. L., Silva, R. C. R. D., \& Silva, J. C. N. D. Indicadores antropométricos de excesso de gordura corporal em mulheres. Rev Bras Med Esporte, 12(3), 119-24, 2006.

14. Silva, S. P.; Santos, A. C. S.; Silva, H. M.; Costa, C. L. A.; Nobre, G. C. Aptidão cardiorrespiratória e composição corporal em crianças e adolescentes. Motriz, Rio Claro, v. 16, n. 3, p.664-671, 2010.

15. Delfino,T., Huber, M. P. Níveis de força/resistênc ia abdominal e índice de massa corporal em alunos de 12 a 15 anos do município de Armazém, SC, Brasil. EFDesportes.com, Revista Digital. Buenos Aires, Año 17, No 178, março de 2013.

16. Youdas, J. W., Krause, D. A., Hollman, J. H., Harmsen, W. S., \& Laskowski, E. The influence of gender and age on hamstring muscle length in healthy adults. Journal of Orthopaedic \& Sports Physical Therapy, 35(4), 246-252, 2005

17. Youdas, J. W., Krause, D. A., \& Hollman, J. H. Validity of hamstring muscle length assessment during the sit-and-reach test using an inclinometer to measure hip joint angle. The Journal of Strength \& Conditioning Research, 22(1), 303-309, 2008.
18. Mikkelsson, L. O., Nupponen, H., Kaprio, J., Kautiainen, H., Mikkelsson, M., \& Kujala, U. M. Adolescent flexibility, endurance strength, and physical activity as predictors of adult tension neck, low back pain, and knee injury: a 25 year follow up study. British journal of sports medicine, 40(2), 107113, 2006.

19. Fernandes, M. M.; Penha D. S. G.; Braga F. A. Obesidade infantil em crianças da rede pública de ensino: prevalência e consequências para flexibilidade, força explosiva e velocidade. Rev Educ Fís. v, 23, n.4 p.629-34. 2012.

20. Vitorino, P.V.O.; Jardim, P. C. B. V.; Sousa, A. L. L.; Santana, S. F.; Barbosa, M. A. Prevalência de estilo de vida sedentário entre adolescentes. Acta Paulista de Enfermagem (Online), v. 28, p. 166-171, 2015.

21. Regis, M.F.; Oliveira, L.M.F.T.; Santos, A.R.M.; Leonildo, A.C.R.; Diniz, P.R.B.; Freitas, C.M.S.M. Urban versus rural lifestyle in adolescents: associations between environment, physical activity levels and sedentary behavior. Einstein (São Paulo). v. 14. n. 4. p. 461-467. 2016.

22. Guedes, A. C. F., Biscuola, A. P., \& Lima, M. C. C. Comparação entre índice de massa corporal e índice de adiposidade coporal em adultos do sexo masculino. RBONE-Revista Brasileira de Obesidade, Nutrição e Emagrecimento, 9(54), 235-242, 2015.

23. De Lacerda, L. R. F., Rodrigues, A. Y. F., da Silva Rocha, M. R., \& Lopes, S. V. M. U. Prevalência de obesidade infantil e sobrepeso em escolares. Revista Interfaces: saúde, humanas e tecnologia. v.2, n.5 novembro 2014.

24. Farias Júnior, J. C.; Silva, K. S. Overweight/obesity in adolescente students from the city of João Pessoa, PB, Brazil: prevalence and association with demographic and socioeconomic factors. Rev Bras Med Esporte. v. 14, p. 104-8.2008.

25. Carneiro, C. D. S., Peixoto, M. D. R. G., Mendonça, K. L., Póvoa, T. I. R., Nascente, F. M. N., Jardim, T. D. S. V., ... \& Jardim, P. C. B. V. Excesso de peso e fatores associados em adolescentes de uma capital brasileira. Revista Brasileira de Epidemiologia, 20, 260-273, 2017.

26. Guedes, D. P.; Guedes, J. E. R. P.; Barbosa, D. S.; De Oliveira, J. A. Atividade física habitual e aptidão física relacionada à saúde em adolescentes. Revista Brasileira de Ciência e Movimento, 10(1), 13-22. 2008.

27. Ebbeling, C. B.; Pawlak, D. B.; \& Ludwig, D. S. Childhood obesity: public-health crisis, common sense cure. The lancet, 360(9331), 473-482. 2002.

28. Carissimi, A., Adan, A., Tonetti, L., Fabbri, M., Paz Hidalgo, M., Levandovski, R., \& Martoni, M. Autoeficácia física associada ao índice de massa corporal em crianças em idade escolar. J Pediatr. V, 93, n. 8, p.64-9.2017.

29. Burgos, M. S.; Reuter, C. P.; Burgos, L. T.; Pohl, H. H.; Paiva, D. N.; Reuter E. M. Aptidão cardiorrespiratória e fatores de risco cardiovasculares: 
um estudo com escolares de Santa Cruz do Sul, RS, Brasil. Rev Epidem Contr Infecção, v. 3, n.4 .2013.

30. De Andrade Gonçalves, E. C., Silva, D. A. S., \& Gimenes, H. E. Prevalence and Factors Associated With Low Aerobic Performance Levels in Adolescents: A Systematic Review. Current Pediatric Reviews, 11, 000-000.2015.

31. Pelegrini, A., Silva, D. A. S., Petroski, E. L., \& Glaner, M. F. Aptidão física relacionada à saúde de escolares brasileiros: dados do projeto esporte Brasil. Rev. bras. med. esporte, 17(2), 92-96.2011.

32. Nascimento, T. B. R., Pereira, D. C., \& Glaner, M. F. Prevalência de indicadores de aptidão física associada à saúde em escolares. Motriz. Journal of Physical Education. UNESP, 387-394. 2010.

33. Pelegrini, A., Minatto, G., Claumann, G., Silva, D., Grigollo, L., Schwinn, F., \& Petroski, É. Aptidão cardiorrespiratória em adolescentes. Revista Andaluza de Medicina del Deporte, 10(3), 152-157. 2017.

34. De Brito, L. B. B., Ricardo, D. R., de Araújo, D. S. M. S., Ramos, P. S., Myers, J., \& de Araújo, C. G. S. Ability to sit and rise from the floor as a predictor of all-cause mortality. Eur J Prev Cardiol. V.21, n.7, p. 892-8. 2014.

35. Kyu, H. H., Bachman, V. F., Alexander, L. T., Mumford, J. E., Afshin, A., Estep, K., Cercy, K.. Physical activity and risk of breast cancer, colon cancer, diabetes, ischemic heart disease, and ischemic stroke events: systematic review and dose-response meta-analysis for the Global Burden of Disease Study 2013. BMJ; 354. 2016.

36. Guissard, N., Duchateau, J. Neural aspects of muscle stretching. Exercise and sport sciences reviews, 34(4), 154-158.2006.

37. Cornbleet, S. L.; Woolsey, N. B. Assessment of hamstring muscle length in school-aged children using the sit-and-reach test and the inclinometer measure of hip joint angle. Physical Therapy, 76(8), 850855.1996.

38. Reid, D. A.; \& McNair, P. J. Passive force, angle, and stiffness changes after stretching of hamstring muscles. Medicine and Science in Sports and exercise, 36(11), 1944-1948.2004.

39. Hinman, M. R. Comparison of thoracic kyphosis and postural stiffness in younger and older women. The spine journal, 4(4), 413-417.2004.

40. Fernandes, M. M., Penha, D. S. G., \& de Assis, F. Obesidade infantil em crianças da rede pública de ensino: prevalência e consequências para flexibilidade, força explosiva e velocidade. Rev Educ Fís. v, 23, n.4 p.629-34. 2012.

41 Andreasi, V., Michelin, E., Rinaldi, A. E. M., \& Burini, R. C. Aptidão física associada às medidas antropométricas de escolares do ensino fundamental. J. Pediatr. vol.86, n.6, pp. 497-502. 2010.

42. KNAPIK, Joseph J. The importance of physical fitness for injury prevention: part 1 . J Spec Oper Med. v.15 n.1, p.123-127. 2015.

43 Mascarenhas, L. P. G., Ferreira, A. B., de Lima, V. D. A., \& Grzelczak, M. T. Estudo comparativo da aptidão física entre crianças de escola pública e particular: uma visão regional. Cinergis. V,14, n.3, p. 157-60. 2014.

44. Petroski, E. L., Silva, A. F. D., Rodrigues, A. B., \& Pelegrini, A. Aptidão física relacionada a saúde em adolescentes brasileiros residentes em áreas de médio/baixo índice de desenvolvimento humano. Revista de Salud Pública, 13, 219-228. 2011. 45. Luguetti, C. N., Ré, A. H. N., \& Böhme, M. T. S. Indicadores de aptidão física de escolares da região. Centro-oeste da cidade de São Paulo. Rev Bras Cineantropom Desempenho Hum. v. 12, n. 5, p.331337. 2010 